\title{
Elastographic presentation of medullary thyroid carcinoma
}

\author{
Massimiliano Andrioli - Pierpaolo Trimboli - Stefano Amendola • \\ Stefano Valabrega $\cdot$ Nobuhiro Fukunari $\cdot$ Moro Mirella $\cdot$ Luca Persani
}

Received: 9 July 2013/Accepted: 12 September 2013/Published online: 25 September 2013

(C) Springer Science+Business Media New York 2013

\begin{abstract}
Aim of the study was to evaluate the elastographic appearance of medullary thyroid carcinoma (MTC) by a retrospective evaluation of 18 nodules histologically proven as MTC. Free-hand qualitative elastography was performed using Hitachi Logos EUB 7500. The elasticity score (ES), was assessed based on a colour elastogram, the blue colour being correlated with hard tissue, red colour with soft tissue, and green with intermediate hardness. Nodules were classified into four classes. A alleged diagnosis of malignancy was assigned to nodules with ES3 or 4 and a presumptive diagnosis of benignity was assigned to nodules with an ES1 or 2. More than half $(55.6 \%)$ of MTCs have a low-intermediate grade of elasticity. The hardest lesions (ES4) were those with ultrasonographic features highly suspicious for malignancy. In conclusion,
\end{abstract}

M. Andrioli $(\varangle) \cdot$ M. Mirella $\cdot$ L. Persani

Division of Endocrine and Metabolic Diseases, Ospedale San

Luca, IRCCS, Istituto Auxologico Italiano, p.le Brescia 20,

20149 Milan, Italy

e-mail: massimoandrioli1 @ virgilio.it

P. Trimboli $\cdot$ S. Amendola

Section of Endocrinology and Diabetology, Ospedale Israelitico, Rome 00148, Italy

S. Valabrega

Department of Medical and Surgical Sciences, Ospedale

S. Andrea, Sapienza University, Rome, Italy

N. Fukunari

Department of Surgery, Showa University School of Medicine,

Yokohama Northern Hospital, 35-1, Chigasaki-cyuo,

Tsuzuki-ku, Yokohama, Japan

L. Persani

Department of Clinical Sciences and Community Health,

Università degli Studi di Milano, 20122 Milan, Italy most of MTCs present an elastographic pattern of benignity. Therefore, qualitative elastography does not add useful information in pointing out MTC on the basis of its hardness. Our data suggest a marginal role for this technique in MTC evaluation.

Keywords Medullary carcinoma - Thyroid . Elastosonography · Elastography

\section{Introduction}

Thyroid nodules are a very common entity, being detectable in up to $67 \%$ of subjects by ultrasonography (US). Approximately $5 \%$ of all thyroid lesions are cancer [1]. Then, the first aim in thyroid nodule evaluation is to exclude malignancy, which includes differentiated thyroid cancer, medullary cancer (MTC) and, more rarely, anaplastic carcinoma and lymphoma [1]. Cytological examination of material obtained by fine-needle cytology, due to its high sensitivity and specificity, is the best single test for differential diagnosis. However, there is a recognized $5 \%$ false-negative rate and it has the disadvantage of being an invasive procedure. Therefore, recently, US elastography (USE), a novel technology based on the elastic property of the tissue, has been added to the diagnostic armamentarium as an accurate, non-invasive predictor of thyroid malignancy. The technique is based on the principle that malignant lesions are harder than benign ones.

Several studies on thyroid USE have been reported since its first evaluation in thyroid context in 2005 and they generally consider USE useful in detecting malignant lesions [2]. Nevertheless, the majority of these studies focused on differentiated papillary carcinoma (PTC), the most common thyroid cancer. On the contrary, USE 

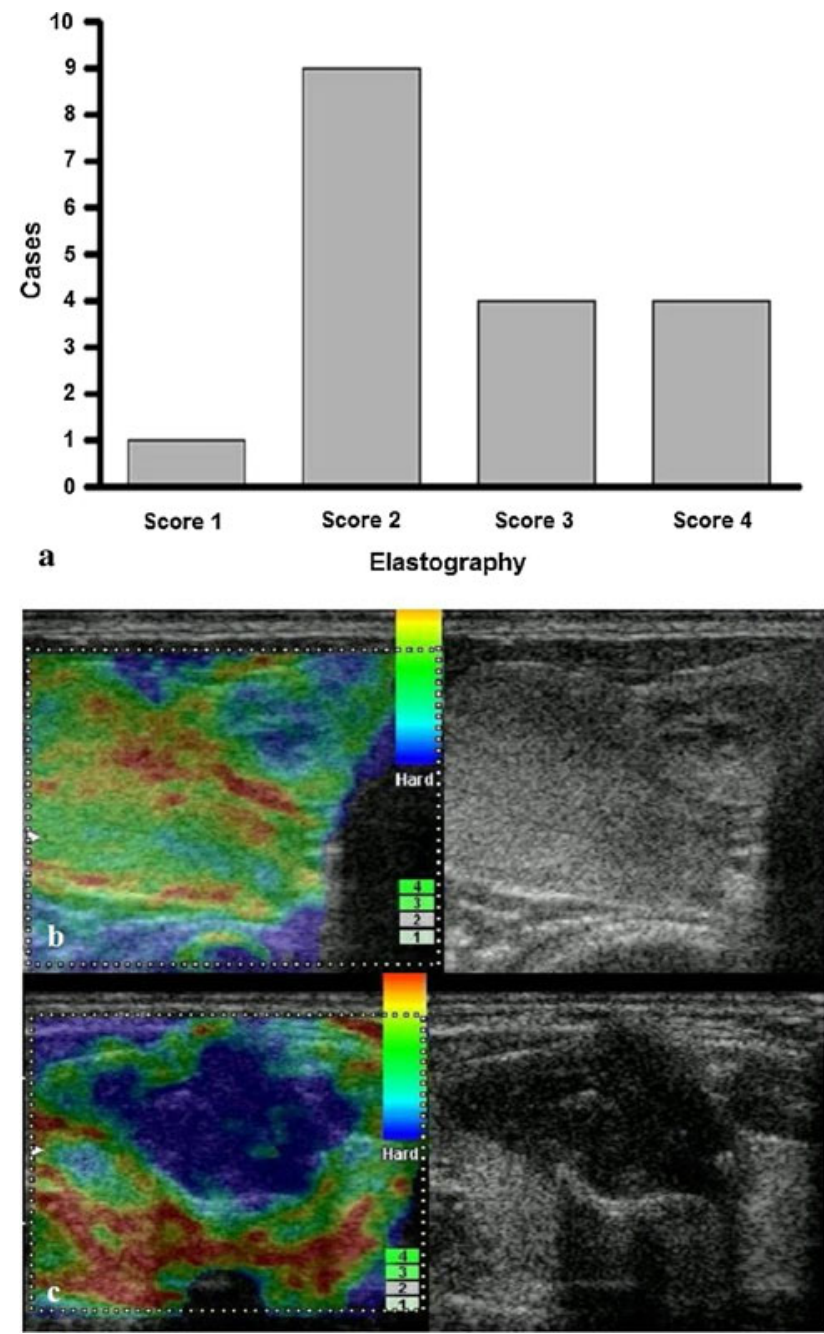

Fig. 1 a The distribution of 18 MTC nodules in the four elastografic classes. b The most frequent elastographic pattern showed by MTC (ES2). This lesion is solid, isoechoic, homogenous and presents regular margins at standard ultrasonography and it appears green in more than half of its surface. c A hard MTC lesion (ES4). The tumour is homogenously blue and therefore suspicious for malignancy. Nevertheless, this lesion was suspicious for malignancy due to its marked hypoechogenicity and the presence of irregular and spiculated margins at standard ultrasound evaluation

features of MTC are incidentally reported only in few reports. In the present study, we examined the elastographic features of MTC and discussed the role of USE in this kind of thyroid cancer.

\section{Patients and methods}

Eighteen consecutive MTCs were retrospectively enrolled from three different Institutes: seven cases at Istituto Auxologico Italiano of Milan, other seven cases at Yokohama Northern Hospital, and four cases at Ospedale Israelitico of Rome. All MTC lesions were assessed by free- hand real-time qualitative USE. Qualitative thyroid USE was performed, before fine-needle cytology and surgery, using Hitachi Logos EUB 7500, and 6-13 MHz linear probes according to the technique previously described [3], by three experienced examiners (MA, PT, NF).

The USE score, i.e. elasticity score (ES), was assessed based on a colour elastogram, the blue colour being correlated with hard tissue, red with soft tissue, and green with intermediate hardness. Nodules were classified into four classes: ES1: lesion homogeneously red or green; ES2: green displayed in more than half of nodule; ES3: blue displayed in more than half of lesion; ES4: nodule homogenously blue [3]. An alleged diagnosis of malignancy was assigned to nodules with ES 3 or 4, and a presumptive diagnosis of benignity was assigned to nodules with an ES1 or 2. One elastogram image for each MTC lesion was chosen, stored and separately blindly evaluated by each operator. Therefore, each MTC elastogram image received three provisional elasticity scores. The final score is assigned on the basis of the prevalent temporary elasticity score. In case of entirely discordant provisional scores, e.g. three different scores, a collegial re-examination of the recorded pictures was foreseen in order to reach a shared ES.

\section{Results}

Owing to the lack of entirely discordant cases a collegial revaluation was never required.

ES was 1 in 1/18 nodules (5.6\%), 2 in 9/18 lesions (50.0\%), 3 in 4/18 nodules (22.2\%), and 4 in the remaining 4 tumours $(22.2 \%)$ (Fig. 1a). The prevalent USE pattern of MTC was ES 2, and according to our criteria, the majority of MTCs (55.6\%) presented an elastographic pattern of benignity (Fig. 1b). Moreover, the hardest lesions (ES4) were those with other ultrasonographic features highly suspicious for malignancy (Fig. 1c).

\section{Discussion}

Due to its ability in documenting hard lesions, USE has been proposed as an useful non-invasive tool in predicting PTC, the most common thyroid tumour, even if classical ultrasound features of malignancy are lacking [2]. Instead, data on the USE features of MTC are lacking, though an early diagnosis is strongly recommended due to its aggressive behaviour. Moreover, the possible pitfalls of cytology [4] and the reported low prevalence of suspicious ultrasound features [5], represent common causes of missed presurgical diagnosis of MTC. Therefore, USE would represent an additional useful tool and we tested its predictive reliability in this scenario. To the best of our 
knowledge, this is the first report focusing on the USE features of MTCs.

The major finding of our study is that more than half of MTCs have a low-intermediate grade of elasticity, in contrast with reports on PTCs [2]. Most of the MTCs present an elastographic pattern of benignity suggesting that most of the MTC lesions are not hard. Indeed, the hardest medullary lesions (ES4) were those with other US features suggestive of malignancy in our series.

The number of samples here examined is limited suggesting the need of largest prospective studies involving subjects bearing also other thyroid malignancies and benignant nodules. Moreover, the use of more precise USE methods, such as the semiquantitative Strain Ratio-USE and the quantitative Shear Wave-USE, may improve the accuracy of USE in this setting [6, 7]. However, because of the lack of data focusing on USE presentation of MTC, the present data represent a novelty on this topic. In conclusion, qualitative-USE does not appear to add useful information and does not overcome the diagnostic limits of US and cytology in MTC [4, 5]. Our results indirectly support the diagnostic importance of routine serum calcitonin measurement in nodular thyroid disease.

Conflict of interest The authors have no conflicts of interest to disclose.

Ethical standard All patients gave their written informed consent according to the each local Ethics Committee, and the guidelines of the Declaration of Helsinki.

\section{References}

1. H. Gharib, E. Papini, R. Valcavi, H.L. Baskin, A. Crescenzi, M.E. Dottorini, D.S. Duick, R. Guglielmi, C.R. Hamilton, M.A. Zeiger,
M. Zini, AACE/AME task force on thyroid nodules, American Association of Clinical Endocrinologists and Associazione Medici Endocrinologi medical guidelines for clinical practice for the diagnosis and management of thyroid nodules. Endocr. Pract. 12(1), 63-102 (2006)

2. P. Trimboli, R. Guglielm, S. Monti, I. Misischi, F. Graziano, N. Nasrollah, S. Amendola, S.N. Morgante, M.G. Deiana, S. Valabrega, V. Toscano, E. Papini, Ultrasound sensitivity for thyroid malignancy is increased by real-time elastography: a prospective multicenter study. J. Clin. Endocrinol. Metab. (2012). doi:10.1210/ jc.2012-2951

3. M. Scacchi, M. Andrioli, C. Carzaniga, G. Vitale, M. Moro, L. Poggi, F. Pecori Giraldi, L.M. Fatti, F. Cavagnini, Elastosonographic evaluation of thyroid nodules in acromegaly. Eur. J. Endocrinol. (2009). doi:10.1530/EJE-09-0558

4. R. Elisei, V. Bottici, F. Luchetti, G. Di Coscio, C. Romei, L. Grasso, P. Miccoli, P. Iacconi, F. Basolo, A. Pinchera, F. Pacini, Impact of routine measurement of serum calcitonin on the diagnosis and outcome of medullary thyroid cancer: experience in 10,864 patients with nodular thyroid disorders. J. Clin. Endocrinol. Metab. 89(1), 163-168 (2004)

5. P. Trimboli, N. Nasrollah, S. Amendola, F. Rossi, G. Ramacciato, F. Romanelli, P. Aurello, A. Crescenzi, O. Laurenti, E. Condorelli, C. Ventura, S. Valabrega, Should we use ultrasound features associated with papillary thyroid cancer in diagnosing medullary thyroid cancer? Endocr. J. 59(6), 503-508 (2012)

6. J. Bamber, D. Cosgrove, C.F. Dietrich, J. Fromageau, J. Bojunga, F. Calliada, V. Cantisani, J.M. Correas, M. D'Onofrio, E.E. Drakonaki, M. Fink, M. Friedrich Rust, O.H. Gilja, R.F. Havr, C. Jenssen, A.S. Klauser, R. Ohliger, A. Saftoiu, F. Schaefer, I. Sporea, F. Piscaglia, EFSUMB guidelines and recommendations on the clinical use of ultrasound elastography. Part 1: Basic principles and technology. Ultraschall. Med. (2013). doi:10.1055/ s-0033-1335205

7. D. Cosgrove, F. Piscaglia, J. Bamber, J. Bojunga, J.M. Correas, O.H. Gilja, A.S. Klauser, I. Sporea, F. Calliada, V. Cantisani, M. D’Onofrio, E.E. Drakonaki, M. Fink, M. Friedrich Rust, J. Fromageau, R.F. Havre, C. Jenssen, R. Ohliger, A. Saftoiu, F. Schaefer, C.F. Dietrich, EFSUMB guidelines and recommendations on the clinical use of ultrasound elastography. Part 2: Clinical applications. Ultraschall. Med. (2013). doi:10.1055/s-0033-1335375 